\title{
Primary mesenchymal chondrosarcoma of the kidney with synchronous implant and infiltrating urothelial carcinoma of the ureter
}

\author{
Hua Xu1, MuMin Shao ${ }^{1}$, HuiLi Sun ${ }^{2}$ and ShunMin $\mathrm{Li}^{2 *}$
}

\begin{abstract}
Primary mesenchymal chondrosarcoma of the kidney is rare, and it shows distinct undifferentiated tumor cells and well differentiated cartilagenous components. Also assident infiltrating urothelial carcinoma of the ureter is an extremely rare cancer. We report a case of primary mesenchymal chondrosarcoma occurring in the left kidney with an ipsilateral and distinct distal ureteric implant, and a coexisting infiltrating urothelial carcinoma of the ureter in a 64-year-old man. Histopathological examination and immunohistochemical studuies showed the classic features of mesenchymal chondrosarcoma in kidney, as well as a few infiltrating urothelial in ureter. Multitarget fluorescence in situ hybridization (FISH) suggested that the development of the urothelial carcinoma in the ureter may be triggered or induced by the chondrosarcoma component. The patient died 2 month after left nephro-ureterectomy. This is the first reported case of a primary mesenchymal chondrosarcoma of the kidney with coexisting infiltrating urothelial carcinoma of the ureter.
\end{abstract}

Virtual Slides: The virtual slide(s) for this article can be found here: http://www.diagnosticpathology.diagnomx.eu/ vs/1522835667751019

Keywords: Mesenchymal chondrosarcoma, Urothelial carcinoma, Kidney, Ureter

\section{Background}

Mesenchymal chondrosarcoma was first reported by Lichtenstein and Bernstein in 1959 [1]. This malignant tumor mostly arises in the skeleton, and one third of the cases arise in the soft tissue and other organs. Extraskeletal mesenchymal chondrosarcoma is rare, and uaually arises in the head and neck region, followed by the lower extremity, the trunk and the retroperitoneum. Primary mesenchymal chondrosarcoma of the kidney is extremely rare, and the first case was published 25 years ago by Malhotra [2]. To date, there are only seven cases of primary renal chondrosarcoma reported in the English literature [2-7]. We report another case of mesenchymal chondrosarcoma of the kidney with synchronous implant and a coexistent infiltrating urothelial carcinoma of the ureter firstly.

\footnotetext{
*Correspondence: shunminli@126.com

${ }^{2}$ Departments of Nephrology, Shenzhen Affiliated Hospital, Guangzhou

University of Traditional Chinese Medicine, Shenzhen, China

Full list of author information is available at the end of the article
}

\section{Case presentation}

A 64-year-old man presented with gross hematuria and vague pain in the left loin. His medical history was unremarkable. Physical examination revealed percussion pain over the left kidney region. Urinalysis showed positive for protein and red blood cells. Abdominal B ultrasonography revealed left hydronephrosis and a hypoechoic mass in the inferior segment of the left ureter. Magnetic resonance imaging (MRI) confirmed left hydronephrosis, a low signal mass in the upper pole of the kidney, and another mass with high T1, low T2 signal in the inferior segment of the left ureter. Imaging examination showed no abnormality elsewhere. A clinical diagnosis of malignancy of the left kidney and the ureter was made, for which a left nephro-ureterectomy was performed.

Gross examination of the nephro-ureterectomy specimen showed pale grayish solid tumor in the enlarged kidney, and the tumor measured $11 \times 8 \times 6 \mathrm{~cm}$, causing dilatation of the renal pelvis. The tumor invaded the cortex, medulla, adeps renis and involved $90 \%$ of the total kidney, resulting in distortion (Figure 1a). And a 


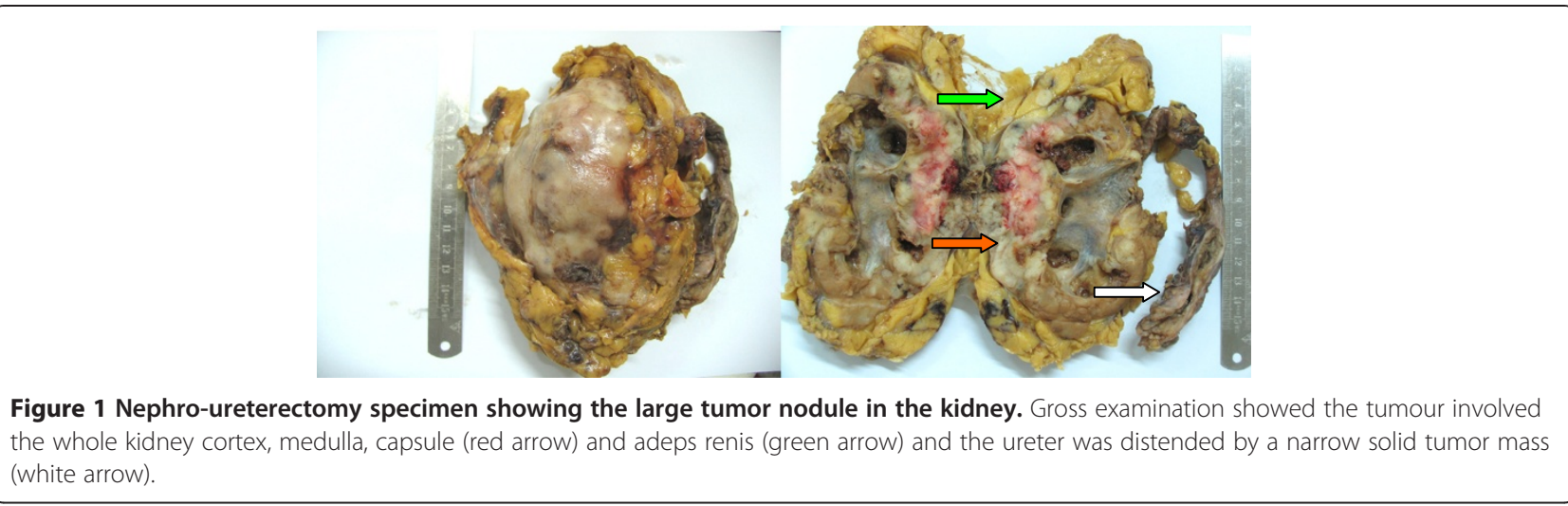

separate elongated solid tumor mass, $7 \times 1.2 \mathrm{~cm}$ in size, was present in the inferior segment of the left ureter, being distinct from the tumor in the renal (Figure 1b). Microscopically, the renal mass showed a biphasic infiltrative growth pattern with the typic characteristic of mesenchymal chondrosarcoma. It consisted of undifferentiated spindle to oval shaped cells, with hyperchromatic nuclei and scanty cytoplasm mainly arranged in Ewing's sarcoma-like, lamellar or hemangiopericytomalike patterns. Other areas demonstrated well defined islands of cartilage. The transformation from the undifferentiated cells to the cartilage cell was abrupt and interlaced(Figure 2a,2b). The tumor had invaded the renal pelvis, but there were no evidence of papillary lesion or underlying urothelial carcinoma.

The tumor in the distal ureter was microscopically similar to that in the kidney, with the typical biphasic mesenchymal chondrosarcoma component, which constituted bulk of the tumor mass. However, there was a small component exhibiting features of urothelial carcinoma. The urothelial carcinoma showed infiltrative nests, of moderate cytological grade, and displayed foci of squamous cell differentiation with some degree of keratinization (Figure 3a,3b). The mucosa adjacent to the tumor showed no evidence of underlying dysplasis or carcinoma in-situ. Extensive sampling of the kidney tumor did not identify a carcinomatous component. Vascular invasion of the chondrosarcoma was detected, but no carcinomous metastasis was detected.

Immunohistochemical staining showed the poordifferentiated tumor cells and cells within the cartilaginous areas in both the kidney (Figure $2 \mathrm{c}, 2 \mathrm{~d}, 2 \mathrm{e}, 2 \mathrm{f}$ ) and the ureter were positive for Vimentin, CD99and S-100 protein but were negative for cytokeratin, epithelial membrane antigen ,E-cadherin, Leu7, neuron-specific enolase, SMA, CD34, p63 and desmin. The poor-differentiated tumor cells displayed a higher Ki-67 index than those in the well-differentiated cartilaginous areas. The urothelial carcinoma component within the ureter, the tumor cells were positive for cytokeratin (Figure 3c), epithelial membrane antigen, E-Cadherin and negative for Vimentin (Figure 3d), S-100 and CD99, enhancing the contrast of synchronous urothelial carcinoma from mesenchymal chondrosarcoma. For FISH analysis, labeled probes specific for chromosomes 3, 7, 17 and for the p16 (9p21) gene (GP Medical Technologies,Ltd, Beijing, China) were used. Two DNA- probes were mixed together as a set double-target FISH and paired as follows: chromosome 3 (fluorescein isothiocyanate) and chromosome 7 (rhodamine), chromosome 17 (fluorescein isothiocyanate) and p16 (rhodamine).In both the urothelial carcinoma and mesenchymal chondrosarcoma components, aneuploidy of chromosome 3, 7 and 17 and loss of p16 gene were observed (Figure 4, and Tables 1,2).

The patient died of the widespread metastases two months after surgery, autopsy was not performed.

\section{Conclusions}

On review of the literature, only seven cases of renal mesenchymal chondrosarcoma have been reported. Here we first describe an interesting case of renal mesenchymal chondrosarcoma with a synchronous ureter implant, which was intimately mixed or in collision with an urothelial carcinoma. The biphasic growth pattern of highly cellular undifferentiated tumor cells, with islands of differentiated cartilage is rather characteristic of mesenchymal chondrosarcoma [8]. Such a distinctive morphology, with the absence of osteoid formation set mesenchymal chondrosarcoma apart from small cell osteosarcoma, Ewing's sarcoma, dedifferentiated chondrosarcoma, and hemangiopericytoma.

It is important to note that the absence of epithelial component and of an underlying urothelial carcinoma from an extensively sampled tumor, is an indication of pure mesenchymal chondrosarcoma arising from the kidney. The lack of immunoreactivity for epithelial markers, suck as cytokeratin AE1/E3, EMA and E-cadherin lends further support to it. Moreover, the tumor located in the renal parenchyma and distant inferior ureter, not in the calyx and pelvis, the relatively normal collecting 

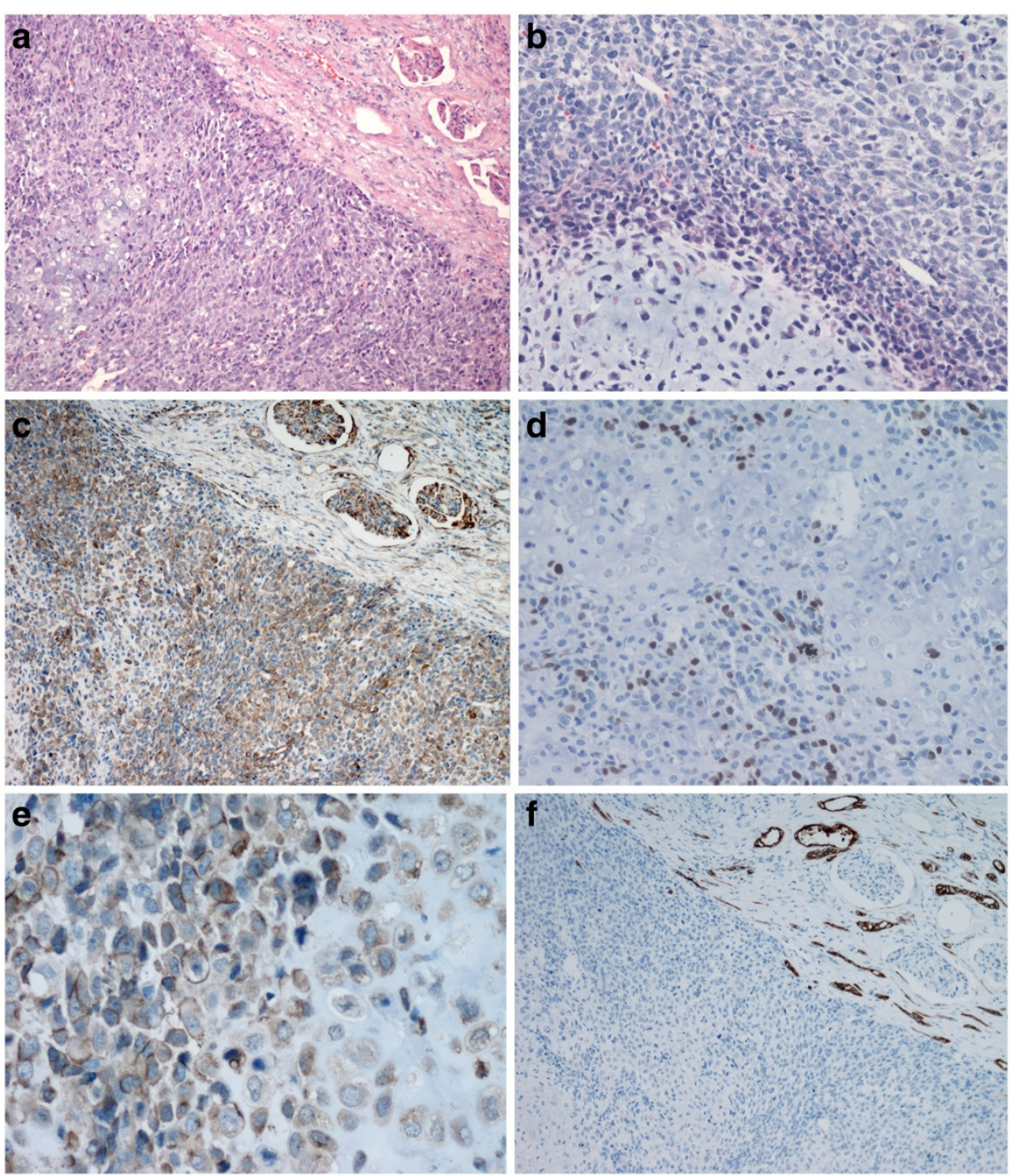

Figure 2 The renal mass consisting of sheets of undifferentiated mesenchymal cells (H\&E, 2a), and scattered well demarcated islands of differentiated cartilage (H\&E, $\mathbf{2 b}$ ). Both the undifferentiated tumor cells and cartilaginous islands were immunoreactive to Vimentin (2c), to S-100 protein (2d) and to CD99 (2e), and not to cytokeratin (2f).

system and urothelium that separates the renal tumor and the distal ureteric mesenchymal chondrosarcoma is keeping with the label of tumor implant.

As for the lower ureteric tumor, the transition between the large bulk of mesenchymal chondrosarcoma and the relatively small urothelial carcinoma was abrupt. Moreover, there was no apparent transition between the carcinoma and the ureteric mucosa or epithelium, which is free of dysplasia or neoplastic field changes. Such an observation support a collision of two separate tumors in the lower ureter. There were some reports on a collision of separate tumors in the same location of the urinary system $[9,10]$. While only vascular invasion is observed with mesenchymal chondrosarcoma, and no observed with urothelial carcinoma, such a pattern of metastasis does not necessarily imply that the two malignancies are biologically distinct.

From the view of histogenesis, some groups have proposed that a cell could generate a precursor with greater potential than the cell from which it derives, or transdifferentiation, that allows cell lineage switch. Several such multipotential malignant sarcoma have been reported, for example osteosarcoma and Ewing's sarcoma with epithelial differentiation [11]. The primary tumor cell in mesenchymal chondrosarcoma represents a very primitive mesenchymal cell type. Daniel Rubio clearly demonstrated a novel mesenchymal-epithelial transition (MET) associated epithelial tumorigenesis [12]. And a work showing MET in vitro using a chondrosarcoma tumor cell line has been reported. Fitzgerald MP reported 


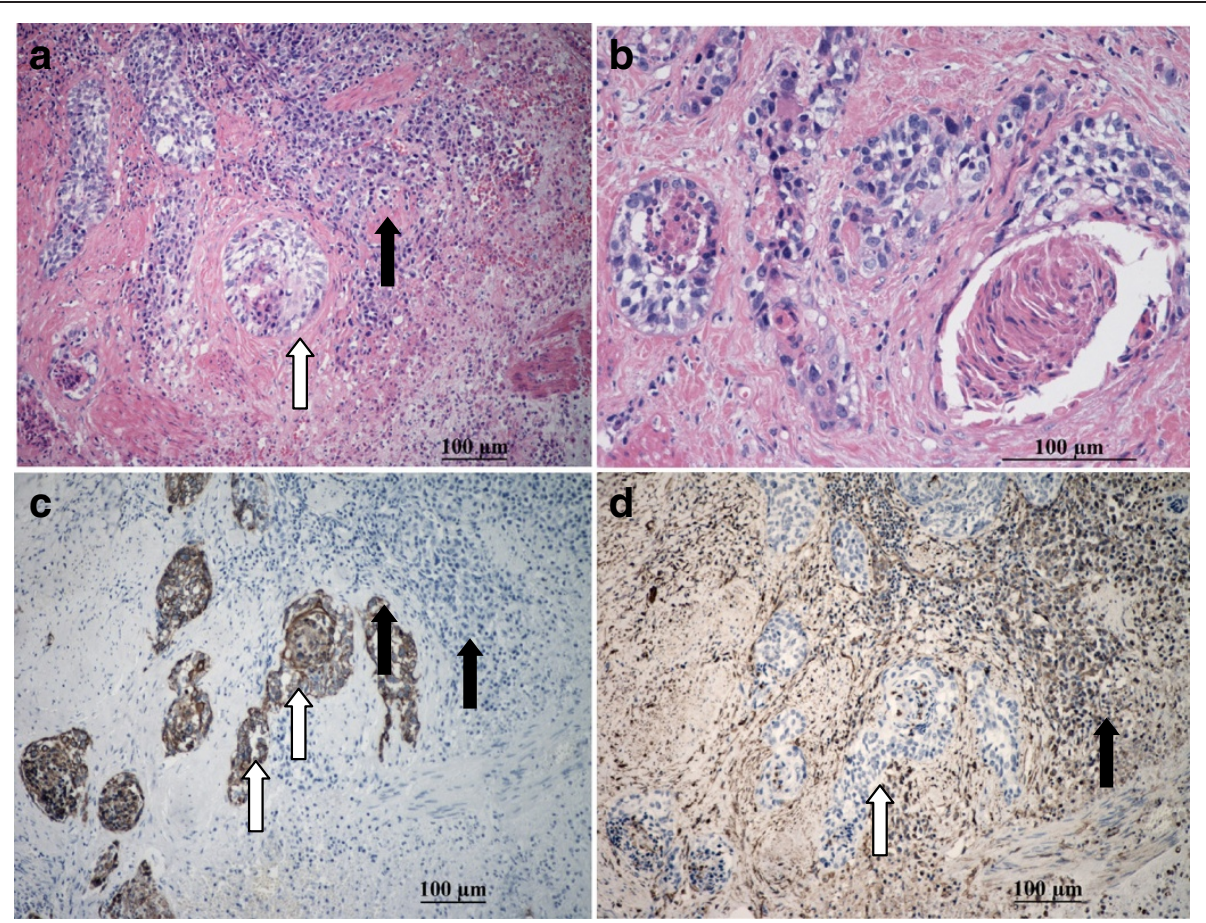

Figure 3 The ureteric tumor revealing a mesenchymal chondrosarcoma, similar to that in the kidney (black arrow), and in addition a synchronous infiltrative urothelial carcinoma (white arrow) (3a), with foci of squamous differentiation and keratinization(3b). The urothelial carcinoma (white arrow) was positive for cytokeratin (3c) and negative for Vimentin (3d); while the mesenchymal chondrosarcoma (black arrow) was negative for cytokeratin (3c) and positive for Vimentin (3d).

Chondrocytes are mesenchymally derived cells that reportedly acquire some epithelial characteristics, and there is an epigenetic switch associated with an METlike phenomenon that accompanies chondrosarcoma progression [13]. These observations support the hypothesis that not all carcinomas are derived from epithelial cells. In our present case, both the urothelial carcinoma and the mesenchymal chondrosarcoma all exhibited polysomy of chromosome 3,7 and 17 , the difference was significant $(\mathrm{p}<0.05$, chi-square test, SPSS 13.0), and loss of p16 gene by fluorescence in situ hybridization (FISH). The development of urothelial carcinoma had been linked to chromosomal instability, especialy chromosome 3,7 and 17 and partial or complete loss of chromosome 9 ( p16 locus) [14] . It is thus conceivable that the development of urothelial carcinoma may be triggered or induced by chondrosarcoma, especially since both shared the same clonal origin.

The cytogenetic and molecular mechanism on renal mesenchymal chondrosarcoma is indefinite. Lee AF showed that, in contrast to Ewing sarcoma, small cell osteosarcoma and mesenchymal chondrosarcoma lack
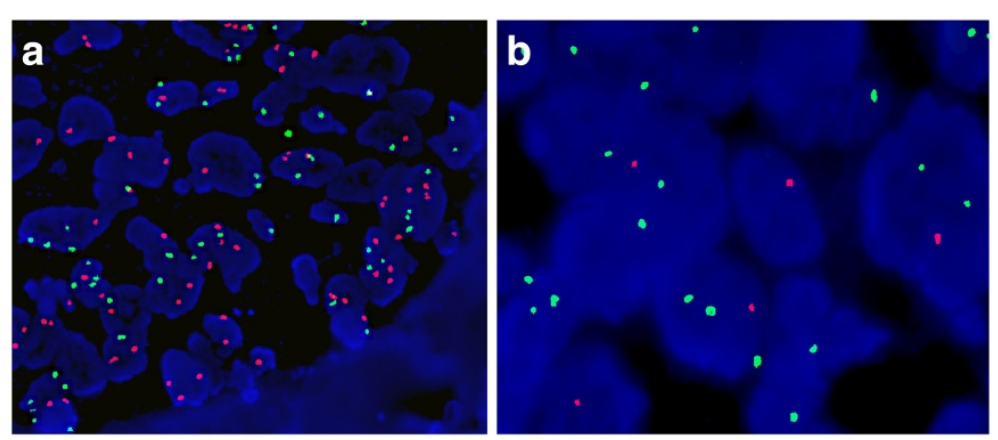

Figure 4 Aneuploidy of chromosome $\mathbf{3}$ (green) and $\mathbf{7 ( r e d )}$ in urothelial carcinoma (4a). Aneuploidy of chromosome17(green)and loss of the p16 gene(red) in urothelial carcinoma (4b). 
Table 1 The ratio of gene copy numbers to chromosome 3,7and 17 centromere by FISH probes in urothelial carcinoma and mesenchymal chondrosarcoma

\begin{tabular}{|c|c|c|c|c|c|c|}
\hline \multicolumn{4}{|c|}{ Urothelial carcinoma } & \multicolumn{3}{|c|}{ Mesenchymal chondrosarcoma } \\
\hline chromosome & 3 & 7 & 17 & 3 & 7 & 17 \\
\hline monosomy(\%) & 8 & 15 & 8 & 12 & 13 & 15 \\
\hline disomy(\%) & 35 & 42 & 29 & 51 & 61 & 46 \\
\hline trisomy(\%) & 27 & 23 & 40 & 18 & 16 & 28 \\
\hline polysomy(\%) & 30 & 20 & 23 & 19 & 10 & 11 \\
\hline aneuploidy(\%) & 65坋 & $58 \triangle$ & $71 \diamond$ & $49 \varsigma$ & $39 \triangle$ & $54 \diamond$ \\
\hline
\end{tabular}

Relationship between aneuploidy of chromosome 3,7, 17 of urothelial carcinoma and mesenchymal chondrosarcoma. 站 $x^{2}=5.22, \mathrm{p}=0.022$. $\Delta x^{2}=7.23, p=0.007 . \diamond x^{2}=6.17, p=0.013$.

FLI-1(Friend leukemia virusintegration-1) immunoreactivity. FLI-1 is therefore useful in the differential diagnosis of small round blue cell tumors of the bone [15]. Wang L described the novel HEY1-NCOA2() fusion appears to be the defining and diagnostic gene fusion in mesenchymal chondrosarcomas [16]. Nevertheless, the progression mechanism of renal mesenchymal chondrosarcoma remains to be developed investigated.

The prognosis for mesenchymal chondrosarcoma arising from bone and soft tissue is dismal. Of the seven cases of primary renal mesenchymal chondrosarcoma reported in the English literature, four cases developed local recurrences or metastases at an interval of 1 month to 2.5 years after surgical intervention. In the current case, whether the ureteric tumor represented a direct intraluminal seeding and 'implant', or a second primary arising from a background with field change remains speculative, but given its highly aggressive nature, it would be tempting to postulate that this highly aggressive tumor shows a propensity for intra-calyxceal spread. The autopsy was not performed, however vascular mesenchymal chondrosarcoma metastasis detected may implicate mesenchymal chondrosarcoma, rather than carcinoma as the lethal metastasis.

\section{Consent}

Written informed consent was obtained from the next of kin of the patient for publication of this Case Report

Table 2 Gene copy numbers of the p16 (9p21) gene by FISH probe

\begin{tabular}{lll}
\hline Urothelial carcinoma(\%) & $\begin{array}{l}\text { Mesenchymal } \\
\text { chondrosarcoma(\%) }\end{array}$ \\
\hline single signal & 36 & 46 \\
\hline double signals & 39 & 39 \\
\hline three signals & 0 & 0 \\
\hline no signal & 25 & 15 \\
\hline gene loss & 61 & 61 \\
\hline
\end{tabular}

The ratio of the loss of $\mathrm{p} 16$ gene are equal. and any accompanying images. A copy of the written consent is available for review by the Editor-in-Chief of this journal.

\section{Abbreviations \\ MRI: Magnetic resonance imaging; Sox-9: Sex determining region Y-box 9; FISH: Fluorescence in situ hybridization; FLI-1: Friend leukemia virusintegration-1; MET: Mesenchymalto epithelial transition.}

\section{Competing interests}

The authors have no competing interests.

\section{Authors' contributions}

HX carried out the molecular genetic studies and participated in draft the manuscript. MMS carried out the immunoassays, participated in the design of the study, participated in draft the manuscript and performed the figures and statistical analysis. HLS participated in its design and clinical data collection, helped in statistical analysis. SML coordination and helped to draft the manuscript. All authors read and approved the final manuscript.

\section{Author details}

${ }^{1}$ Departments of Pathology, Shenzhen Affiliated Hospital, Guangzhou University of Traditional Chinese Medicine, Shenzhen, China. ${ }^{2}$ Departments of Nephrology, Shenzhen Affiliated Hospital, Guangzhou University of Traditional Chinese Medicine, Shenzhen, China.

Received: 19 June 2012 Accepted: 17 September 2012

Published: 21 September 2012

\section{References}

1. Lichtenstein L, Bernstein D: Unusual benign and malignant chondroid tumors of bone. Cancer 1959, 12:1142-1157.

2. Malhotra CM, Doolittle CH, Rodil JV, Vezeridis MP: Mesenchymal chondrosarcoma of the kidney. Cancer 1984, 54:2495-2499.

3. Pitfield J, Preston BJ, Smith PG: A calcified renal mass: chondrosarcoma of kidney. Br J Radiol 1981, 54:262.

4. Nativ O, Horowitz A, Lindner A, Many M: Primary chondrosarcoma of the kidney. J Urol 1985, 134:120-121.

5. Gomez-Brouchet A, Soulie M, Delisle M-B, Escourrou G: Mesenchymal chondrosarcoma of the kidney. J Urol 2001, 166:2305.

6. Kaneko T, Suzuki Y, Takata R, Takata K, Sakuma T, Fujioka T: Extraskeletal mesenchymal chondrosarcoma of the kidney. Int J Urol 2006, 13:285-286

7. Buse S, Behnisch W, Kulozik A, Atschbach F, Hohenfellner M: Primary chondrosarcoma of the kidney. Urol Int 2009, 83:116-118.

8. Shakked RJ, Geller DS, Gorlick R, Dorfman HD: Mesenchymal chondrosarcoma: clinicopathologic study of 20 cases. Arch Pathol Lab Med 2012, 136(1):61-75.

9. Armah HB, Parwani AV, Perepletchikov AM: Synchronous primary carcinoid tumorand primary adenocarcinoma arising within mature cystic teratomao fhorseshoe kidney: a unique case report and review of the literature. Diagn Pathol 2009, V4N:17.

10. Funez R, Pereda T, Rodrigo I, Robles L, Gonzalez C: Simultaneous chromophobe renal cell carcinoma and squamous renal cell carcinoma. Diadn Pathol 2007, V2N:30.

11. Shiraishi J, Mukal M, Yabe H, Shibata R, Yamada T, Miura K, Anazawa U, Morioka H, Sakamoto M: Primary bone carcinosarcoma:chondrosarcoma and squamous cell carcinoma with keratin pearl formation. Path Int 2005, 55:504-509.

12. Daniel R, Silvia G, De la Cueva T, Paz MF, Lloyd AC, Antonio B, Javier G-C: Human mesenchymal stell cell transformation is associated with a mesenchymal-epithelial transition. Exp Cell Res 2008, 314:691-698.

13. Fitzgerald MP, Gourronc F, Teoh ML, Provenzano MJ, Case AJ, Martin JA, Domann FE: HumanChondrosarcomaCellsAcquireanEpithelial-Like Gene Expression Pattern via an Epigenetic Switch: Evidence for MesenchymalEpithelial Transition during Sarcomagenesis. Sarcoma 2011, 2011:598218. Epub 2011 Mar 17.

14. Luo B, Li W, Deng CH, Zheng FF, Sun XZ, Wang DH, Dai YP: Utility of fluorescence in situ hybridization in the diagnosis of upper urinary tract urothelial carcinoma. Cancer Genet Cytogenet 2009, 189(2):93-97. 
15. Lee $A F$, Hayes MM, Lebrun D, Espinosa I, Nielsen GP, Rosenberg AE, Lee $C H$ : FLI-1 distinguishes Ewing sarcoma from small cell osteosarcoma and mesenchymal chondrosarcoma. Appl Immunohistochem Mol Morphol 2011, 19(3):233-238.

16. Wang L, Motoi T, Khanin R, Olshen A, Mertens F, Bridge J, Dal Cin P, Antonescu CR, Singer S, Hameed M, Bovee JV, Hogendoorn PC, Socci N, Ladanyi M: Identification of a novel, recurrent HEY1-NCOA2 fusion in mesenchymal chondrosarcoma based on a genome-wide screen of exon-level expression data.Genes Chromosomes. Cancer 2012, 51(2):127-139.

doi:10.1186/1746-1596-7-125

Cite this article as: Xu et al:: Primary mesenchymal chondrosarcoma of the kidney with synchronous implant and infiltrating urothelial carcinoma of the ureter. Diagnostic Pathology 2012 7:125.

\section{Submit your next manuscript to BioMed Central and take full advantage of:}

- Convenient online submission

- Thorough peer review

- No space constraints or color figure charges

- Immediate publication on acceptance

- Inclusion in PubMed, CAS, Scopus and Google Scholar

- Research which is freely available for redistribution 